\title{
Iron- and Silicon-Intercalated Graphene on Silicon Carbide: from Hybridization to Quasi-Freestanding Bilayer Graphene
}

\author{
E.Yu. Lobanova ${ }^{1}$, E.K. Mikhailenko ${ }^{2,3}$ and G.S. Grebenyuk ${ }^{4}$ \\ ${ }^{1}$ School of Photonics, ITMO University, Kronverkskiy pr., 49, lit. A, St. Petersburg, 197101, Russia \\ ${ }^{2}$ Saint Petersburg Electrotechnical University “LETI”, Professora Popova st., 5, 197376, St. Petersburg, Russia \\ ${ }^{3}$ Petersburg Nuclear Physics Institute named by B.P. Konstantinov of National Research Centre «Kurchatov Institute», \\ mkr. Orlova roshcha, 1, 188300, Gatchina, Leningradskaya oblast, Russia \\ ${ }^{4}$ Plasma Physics, Atomic Physics and Astrophysics Division, Ioffe Institute, Politekhnicheskaya st., 26, \\ St. Petersburg, 194021, Russia
}

Received: December 15, 2020

\begin{abstract}
The intercalation approach has been used for the synthesis of the ultrathin films of iron and its silicides under graphene grown on silicon carbide. Experiments have been carried out under ultrahigh vacuum conditions. The use of low energy electron diffraction together with photoelectron spectroscopy allowed to trace chemical state, elemental composition, and structural changes of the samples at all stages of synthesis. The optimal conditions of iron and silicon intercalation are realized in the temperature range of $400-500{ }^{\circ} \mathrm{C}$. Subsequent intercalation of graphene with $\mathrm{Fe}$ and $\mathrm{Si}$ leads to the formation of $\mathrm{Fe}-\mathrm{Si}$ solid solution layer covered with $\mathrm{Fe}_{3} \mathrm{Si}$ surface silicide. First-principles calculations were carried out using density functional theory. It is shown that iron silicide formation is accompanied by the relaxation of the buffer layer and its transformation to the second layer of graphene.
\end{abstract}

\section{INTRODUCTION}

Graphene remains one of the most attractive materials for use in electronics and spintronics devices [1]. One of the popular methods for its synthesis is the thermal decomposition of silicon carbide. This process makes it possible to obtain high-quality graphene on a dielectric substrate. However, it is accompanied by the formation of a buffer layer consisting of carbon atoms and located between graphene and the substrate [2]. The buffer layer is strongly coupled with the substrate, which causes a high rate of carrier scattering and hence negatively affects graphene transport characteristics. A promising way to reduce the interaction between the buffer layer and the substrate is the intercalation process, i.e. introduction of atoms of other substances under graphene. Intercalation can also be a tool for modifying graphene properties and synthesizing new materials. In particular, the intercalation of graphene with magnetic atoms and the formation of graphene/ferromagnet/dielectric struc- tures is one of the possible ways to create spintronic devices based on graphene. Therefore, the present work aims to study graphene/ferromagnet/dielectric structures obtained from graphene grown on silicon carbide via its intercalation with iron and silicon atoms.

\section{EXPERIMENTAL TECHNIQUE}

The experiments were performed using the equipment of the Russian-German beamline at Helmholtz-Zentrum Berlin (BESSY II). The initial samples (graphene-covered $4 \mathrm{H}-\mathrm{SiC}(0001)$ ) were previously prepared as described in [2]. In the beginning, the samples were cleaned by annealing at $500{ }^{\circ} \mathrm{C}$. Intercalation of iron and silicon was performed by thermal deposition of thin films of these materials on the surface of samples with subsequent annealing at various temperatures. The amount of deposited material was controlled using quartz microbalance. All experiments were done in situ under ultrahigh vacuum conditions $\left(P \sim 10^{-10}\right.$ Torr $)$. The

Corresponding author: E.Yu. Lobanova, e-mail: elobanova@itmo.ru 


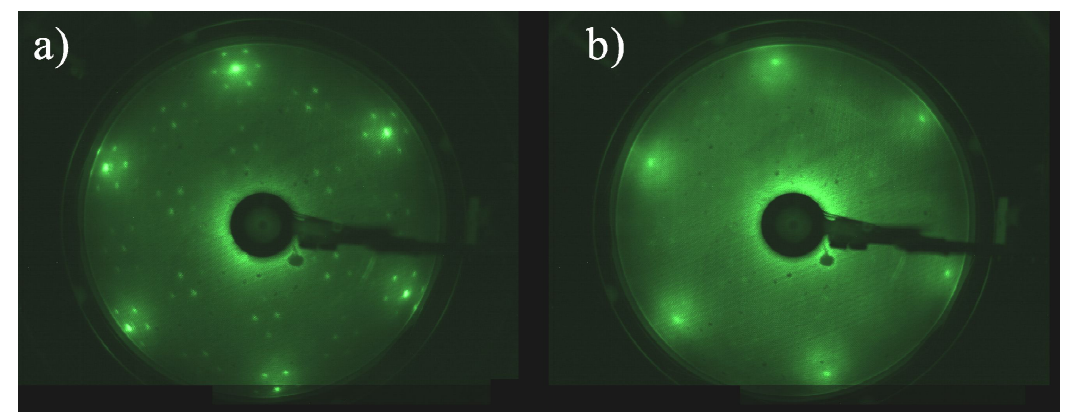

Fig. 1. LEED pattern of the initial graphene/SiC sample (a) and after its intercalation with iron and silicon (b).

characterization of the samples was carried out by low energy electron diffraction (LEED) and photoelectron spectroscopy (PES). The photon energy ranged from 135 to $650 \mathrm{eV}$. The photoelectron spectra were taken using a SPECS PHOIBOS 150 hemispherical energy analyzer.

\section{CALCULATIONS DETAILS}

First-principles calculations were performed in the frame of density functional theory (DFT) using open-source software Quantum ESPRESSO [3]. For the approximation of exchange-correlation potential general gradient approximation (GGA) [4,5] and PAW pseudopotentials [6] were used. The cutoff energy of wave functions was set to 200 Ry. The Monkhorst-Pack mesh of $8 \times 8 \times 1 \mathrm{k}$ points [7] was used for integration over reverse space.

For graphene on silicon carbide, the reconstruction of $p(6 \sqrt{3} \times 6 \sqrt{3}) R 30^{\circ}$, which corresponds to $12 \times 12$ graphene cells, is distinctive. However, modeling such a large cell is resource-intensive. Therefore, in ab initio calculations of the electronic structure of graphene on silicon carbide, a simplified model, where four graphene cells $(2 \times 2)$ are placed on three surface cells of $\mathrm{SiC}$ (0001) $(\sqrt{3} \times \sqrt{3})$, is commonly used [8]. The supercell of the initial system consisted of four $\mathrm{SiC}$ bilayers (three $\mathrm{Si}$ and $\mathrm{C}$ atoms per layer) with graphene ( $8 \mathrm{C}$ atoms) and a buffer layer ( $8 \mathrm{C}$ atoms) on top. The supercell was translated as a hexagonal Bravais lattice with a lattice constant equal to $10.64 \AA$. The thickness of the vacuum gap was $16 \AA$. To construct new supercells, atoms of iron or iron and silicon were added to the original system. The geometry optimization of all systems was carried out taking into account dispersion interaction. The obtained atomic positions were used for self-consistent calculations of the band structure.

\section{RESULTS}

After cleaning the surface of the samples by heating in a vacuum, the samples were characterized. Fig. 1a shows the LEED pattern of graphene/SiC(0001) sample measured at the energy of primary electrons equal to 94 $\mathrm{eV}$. This pattern corresponds to the structure arising from the mismatch of the lattice periods of graphene and silicon carbide.

To control the elemental composition of the samples, survey spectra were measured at a photon energy of $650 \mathrm{eV}$. They showed the presence of only carbon and silicon features, as well as the corresponding peaks of characteristic electron energy losses.

Let us consider the results of graphene intercalation with iron and silicon. First, iron was deposited onto the graphene surface at different substrate temperatures. Then the sample was additionally heated to activate the intercalation process. After that, the same steps were

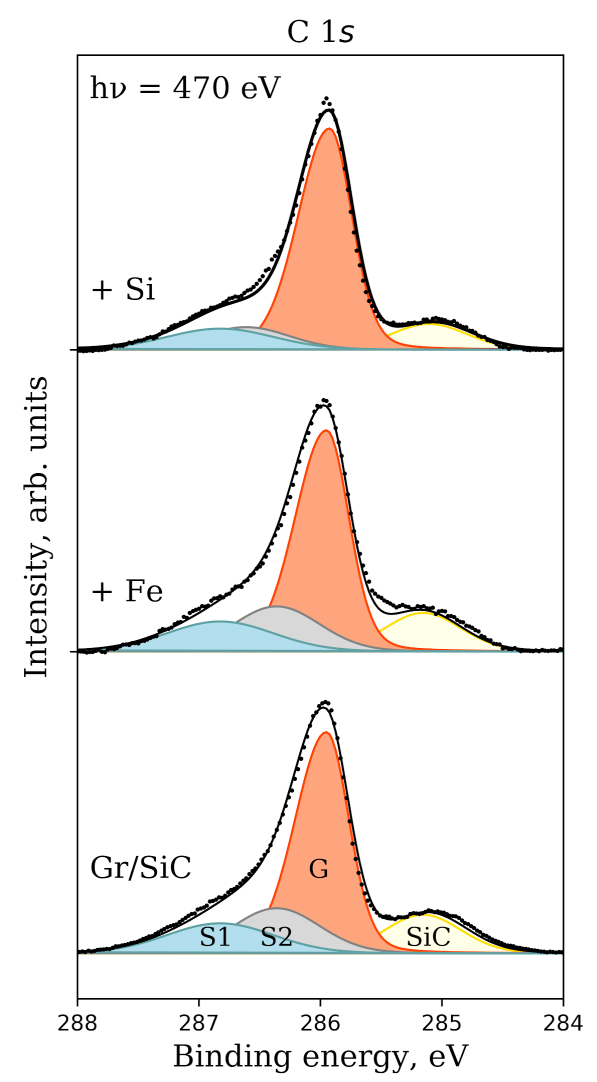

Fig. 2. C $1 s$ spectra on different stages of the system formation. 


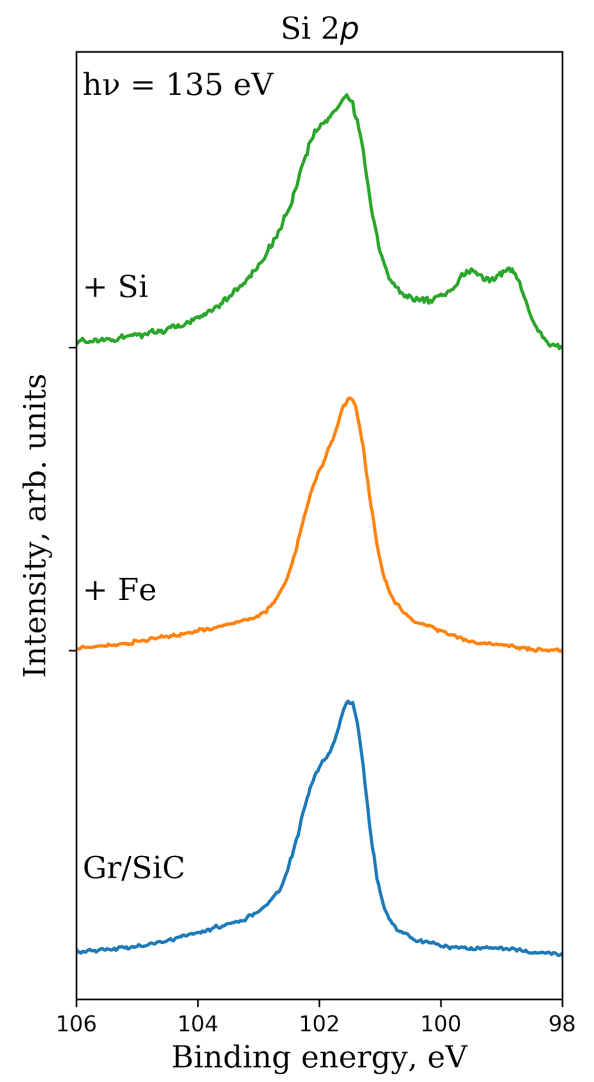

Fig. 3. Si $2 p$ spectra on different stages of system formation.

taken to intercalate silicon. Analysis of the photoelectron spectra showed that the performed annealing led to the penetration of $\mathrm{Fe}$ atoms into the interlayer space between graphene and the substrate. Fig. 2 shows the $\mathrm{C}$ $1 s$ spectra obtained during intercalation of the sample with silicon and iron. The spectrum of the initial system contains modes related to carbon atoms of graphene $(\mathrm{G})$, substrate (SiC), and buffer layer (S1 and S2). The implantation of metal and silicon atoms under graphene leads to attenuation of all spectrum components, except for the graphene mode. This indicates that graphene remains on the surface, and the bonds of the buffer layer with the substrate are broken. Intercalation is possible due to the domain structure of graphene: the diffusion barrier for iron atoms placed on it is low, and at high temperatures metal atoms can migrate to the domain boundaries and penetrate under graphene, forming an iron film under it. The breaking of bonds between the buffer layer and the substrate is evidenced by the LEED patterns. The diffraction pattern observed after iron intercalation (Fig. 1b) indicates the appearance of a thin film between graphene and the substrate, which can be seen from the disappearance of reflexes associated with the reconstruction of the original system. Further intercalation of silicon did not lead to additional changes in LEED patterns.

The change in the $\mathrm{Si} 2 p$ spectra during the intercalation of iron is illustrated in Fig. 3. The interaction of iron with the substrate leads to the appearance of a small feature in this spectrum indicating the beginning of the iron silicides formation. The introduction of silicon atoms leads to the appearance of a mode associated with the formation of $\mathrm{Fe}_{3} \mathrm{Si}$ surface silicide. The optimal conditions for iron and silicon intercalation are realized in the range of $400-500^{\circ} \mathrm{C}$.

In addition to the experimental results, first-principles calculations of the electronic structure of studied intercalation-like systems were carried out. The supercell of the graphene/Fe/SiC(0001) system is shown in Fig. 4a. The distance between the buffer layer and graphene is $3.32 \AA$, and $2.03 \AA$ between the buffer layer and iron. The electronic structure of the system is shown in Fig. 5. The contributions of the $p_{z}$ states of graphene are

a)

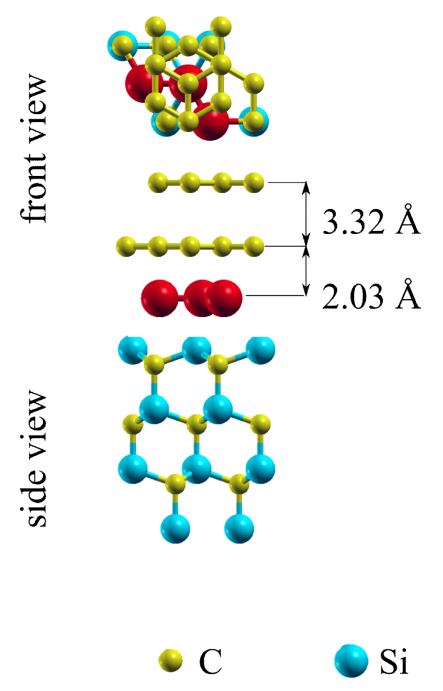

b)

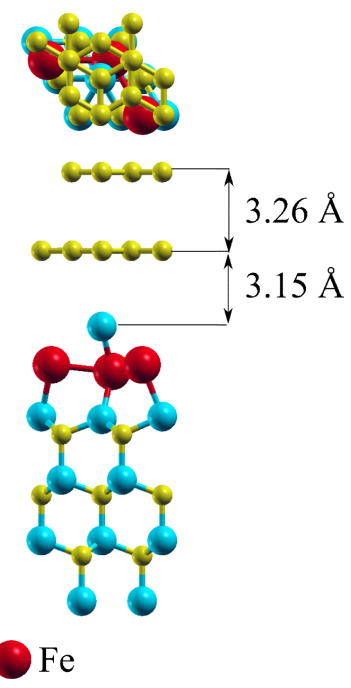

Fig. 4. Supercells used for the graphene/Fe/SiC (a) and the graphene/Si/Fe/SiC simulation. 

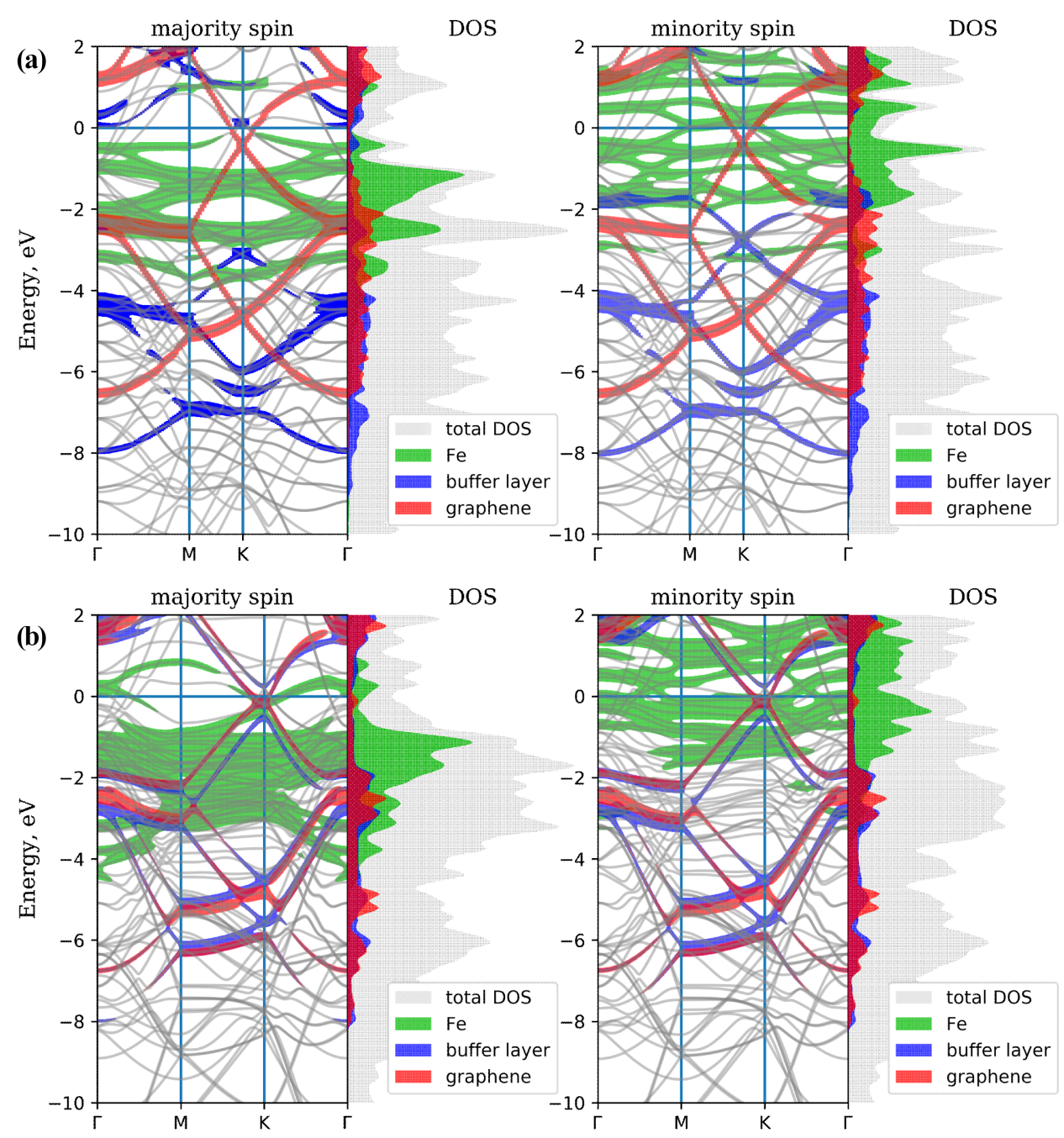

Fig. 5. Electronic structure and corresponding DOS for the graphene $/ \mathrm{Fe} / \mathrm{SiC}$ (a) and the graphene $/ \mathrm{Si} / \mathrm{Fe} / \mathrm{SiC}$ (b) systems.

highlighted in red, the contributions of these states of the buffer layer are shown in blue, and the contributions of the $d$ states of iron atoms are shown in green. The corresponding density of state (DOS) is shown on the side. Due to the spatial overlap of the $p_{z}$ states of the buffer layer carbon atoms and the $d$ states of iron, their hybridization occurs. However, the Dirac cone of graphene exists in the system. It is n-doped and placed $0.5 \mathrm{eV}$ above the Fermi level. The hybridized states of the buffer layer are located $3 \mathrm{eV}$ below the Fermi level. This is consistent with previous calculations for the graphene/Fe/Ni(111) system [9], where strong hybridization affected the graphene electronic structure in the same way. Another effect of hybridization is the spinpolarization of the buffer layer states.

Fig. $4 \mathrm{~b}$ shows a supercell for the graphene $/ \mathrm{Si} / \mathrm{Fe} /$ $\mathrm{SiC}(0001)$ system. The distance between the buffer layer and the substrate turns out to be significantly increased in comparison with the previous case (up to $3.15 \AA$ ), which is due to the incorporation of silicon atoms. Due to the large distance between the buffer layer and the substrate, hybridization does not occur in this case, which leads to the relaxation of the buffer layer. Hence, the buffer layer turns into a second layer of graphene. The corresponding electronic structure is shown in Fig. $5 \mathrm{~b}$. The double paraboloid formed by $p_{z}$ states of graphene and the buffer layer near the $\mathrm{K}$ point is a clear sign of a bilayer graphene electronic structure.

\section{CONCLUSION}

In this work, we studied graphene/ferromagnet/dielectric systems based on graphene grown on $\mathrm{SiC}$. Thin films of iron and its silicides were synthesized under graphene. The optimal temperature for graphene/SiC intercalation with iron and silicon atoms lies in the range of $400-500^{\circ} \mathrm{C}$. The use of high-resolution PES together with LEED made it possible to trace the evolution of the electronic structure of films and their structure in the process of its synthesis. Ab initio calculations of the band structures of the systems under study were carried out. It is shown that metal and silicon atoms during 
intercalation are localized between the substrate and the buffer layer. Intercalation of a metal film is accompanied by a strong hybridization of the states of metals and carbon atoms of the buffer layer. Further introduction of silicon atoms leads to the relaxation of the buffer layer and its transformation into the second graphene layer.It is shown that the electronic structure of all systems is spin-polarized.

\section{ACKNOWLEDGMENTS}

The authors are grateful to V.Yu. Davydov, I.A. Eliseev, S.P. Lebedev, A.A. Lebedev and A.N. Smirnov for the production and certification of graphene/SiC samples; to the data processing center of the NRC "Kurchatov Institute" - PNPI for the resources provided. The work was supported by the Russian-German laboratory at HZB BESSY II.

\section{REFERENCES}

[1] K.S. Novoselov, D.V. Andreeva, W. Ren and G. Shan, Graphene and other two-dimensional materials, Front. Phys., 2019, vol. 14, art. 13301. https://doi.org/10.1007/s11467-018-0835-6

[2] V.Y. Davydov, D.Y. Usachov, S.P. Lebedev, A.N. Smirnov, V.S. Levitskii, I.A. Eliseyev, P.A. Alekseev, M.S. Dunaevskiy, O.Y. Vilkov, A.G. Rybkin and A.A.Lebedev, Study of the crystal and electronic structure of graphene films grown on 6 H-SiC (0001), Semiconductors, 2017, vol. 51, no. 8, pp. 1072-1080. https://doi.org/10.1134/ S1063782617080073

[3] P. Giannozzi, O. Andreussi, T. Brumme, O. Bunau, M.B. Nardelli, M. Calandra, R. Car, C. Cavazzoni,
D. Ceresoli, M. Cococcioni and N. Colonna, Advanced capabilities for materials modelling with Quantum ESPRESSO, J. Phys.: Condens. Matter., 2017, vol. 29, no. 39, art. 465901. https://doi.org/10.1088/0953-8984/21/39/395502

[4] D. Vanderbilt, Soft self-consistent pseudopotentials in a generalized eigenvalue formalism, Phys. Rev. B, 1990, vol. 41, no. 11, pp. 7892-7895. https://doi.org/10.1103/ PhysRevB.41.7892

[5] P.E. Blochl, Generalized separable potentials for electronic-structure calculations, Phys. Rev. B, vol. 41, no. 8, no. 8, pp. 5414-5416. https://doi.org/ 10.1103/PhysRevB.41.5414

[6] J.P. Perdew, J. Chevary, S. Vosko, K.A. Jackson, M.R. Pederson and D. Singh, Atoms, molecules, solids, and surfaces: Applications of the generalized gradient approximation for exchange and correlation, Phys. Rev. B, 1992, vol. 46, no. 11, pp. 6671-6687. https://doi.org/10.1103/ PhysRevB.46.6671

[7] H.J. Monkhorst and J.D. Pack, Special points for Brillouin-zone integrations, Phys. Rev. B, 1976, vol. 13, no. 12, pp. 5188 -5192. https://doi.org/ 10.1103/PhysRevB.13.5188

[8] A. Mattausch and O. Pankratov, Ab initio study of graphene on SiC, Phys. Rev. Lett., 2007, vol. 99, no. 7, art. 076802. https://doi.org/10.1103/ PhysRevLett.99.076802

[9] I.I. Pronin, S.M. Dunaevskii, E.Y. Lobanova and E. K. Mikhailenko, Modification of the electronic structure of graphene by intercalation of iron and silicon atoms, Phys. of the Solid St., 2017, vol. 59, no. 10, pp. 2063-2069. https://doi.org/10.1134/ S1063783417100298 